Electronic Supplementary Information

\title{
Low-Temperature Phase Transformation Accompanied with \\ Charge-Transfer Reaction of Polyiodide Ions Encapsulated in Single-Walled Carbon Nanotubes
}

Yukihiro Yoshida, Yosuke Ishii, ${ }^{*}$ Nao Kato, Canghao Li, and Shinji Kawasaki*

Department of Life Science and Applied Chemistry, Nagoya Institute of Technology, Gokiso-cho,

Showa-ku, Nagoya 466-8555, Japan.

*E-mail: ishii.yosuke@nitech.ac.jp, kawasaki.shinji@nitech.ac.jp; Tel/Fax:+81-52-735-5221 

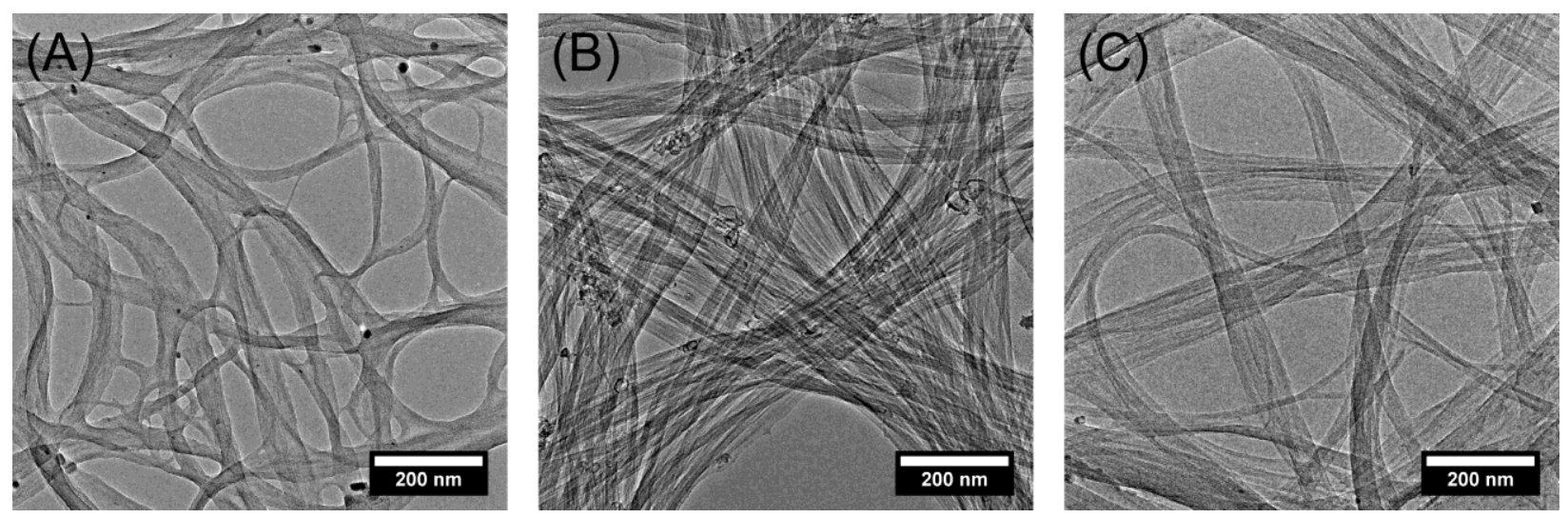

Figure S1. TEM images of (A) HiPco, (B) SO, and (C) EC SWCNTs. 


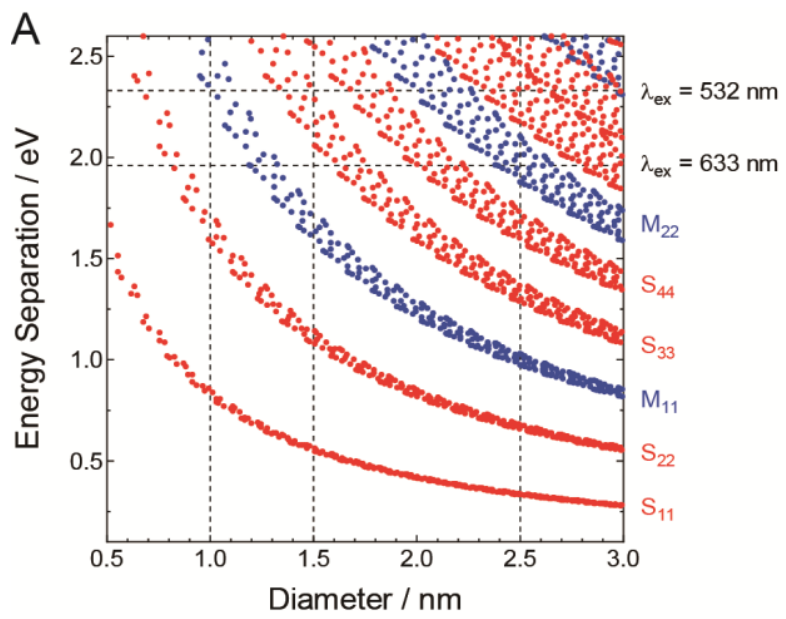

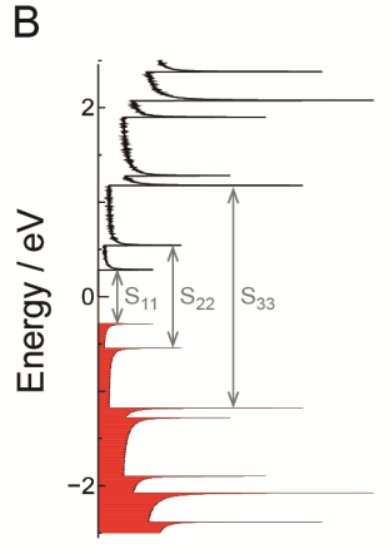

$(17,3) D=1.48 \mathrm{~nm}$

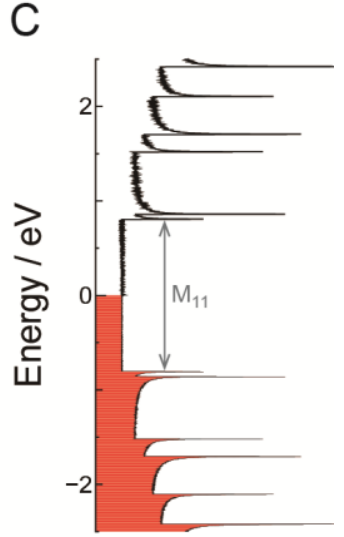

$(15,6) D=1.49 \mathrm{~nm}$

Figure S2. (A) Kataura plot, and electronic density of states (DOSs) of (B) semiconducting and (C) metallic SWCNTs. In the DOS figures, the positions of Fermi energy $\left(E_{\mathrm{F}}\right)$ are set as $0 \mathrm{eV}$ and valence levels are filled with red. 


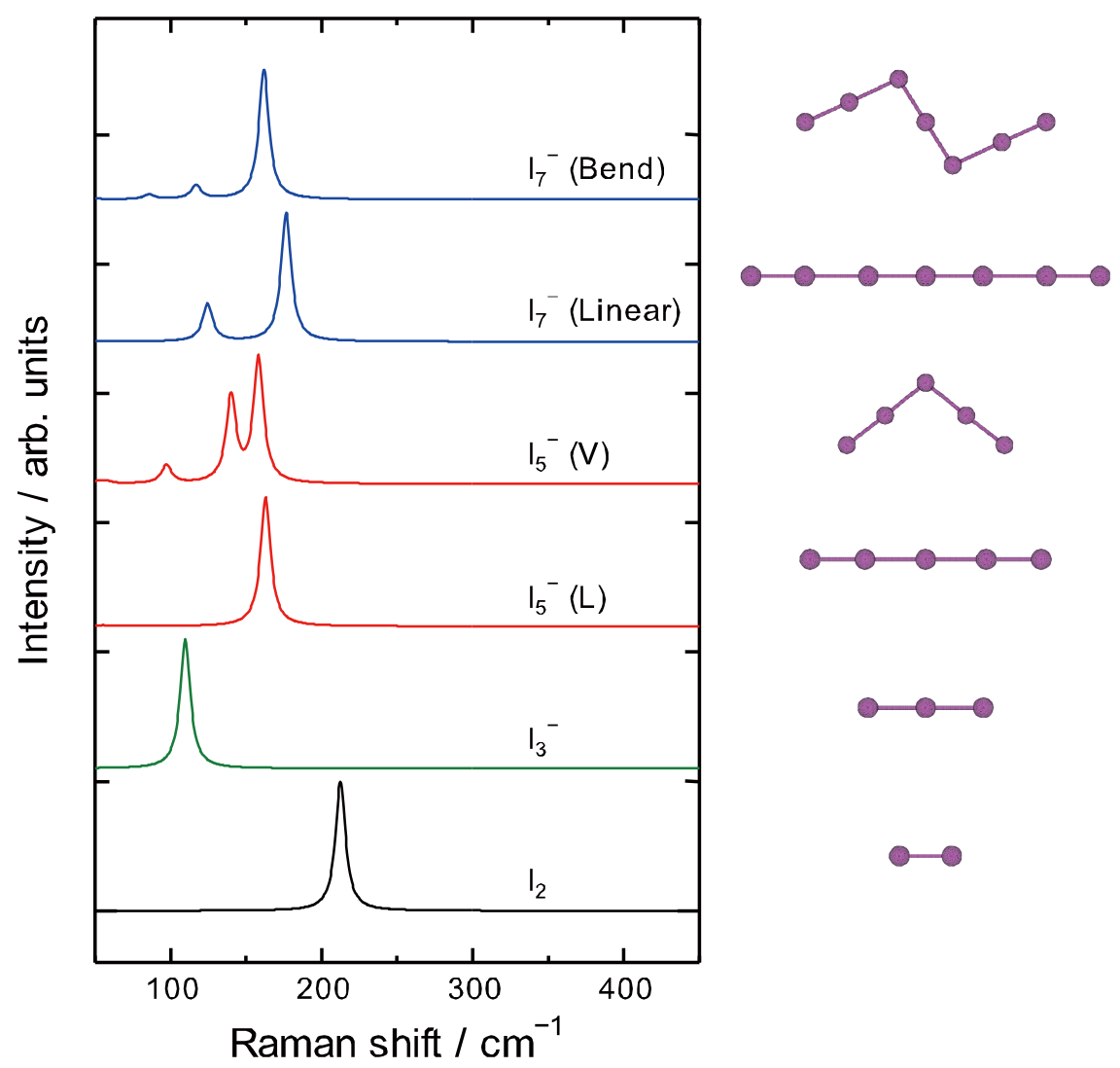

Figure S3. Theoretical Raman spectra of polyiodide ions. 